
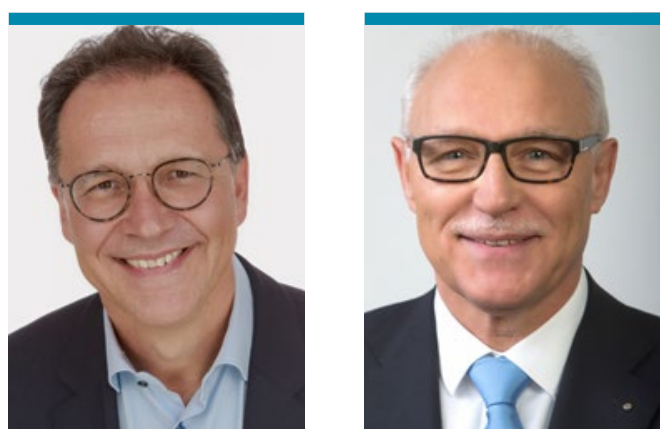

„Sowoh/ Neueinsteiger als auch erfahrene Allergologen

werden vom interaktiven und interdisziplinären Austausch beim 12. Deutschen Allergiekongress profitieren."

Prof. Dr. Ludger Klimek, Zentrum für Rhinologie und Allergologie Wiesbaden

Prof. Dr. Wolfgang Wehrmann, Dermatologische Gemeinschaftspraxis Münster

\title{
Gemeinsam für die Allergologie!
}

$$
\text { L }
$$

iebe Kolleginnen und Kollegen, wir dürfen Sie hiermit herzlich einladen zum 12. Deutschen Allergiekongress (DAK), der vom 5. bis 7. Oktober 2017 in Wiesbaden stattfinden wird.

Mit der gemeinsamen Ausrichtung des DAK zeigen der Ärzteverband Deutscher Allergologen (AeDA), die Gesellschaft für Pädiatrische Allergologie und Umweltmedizin (GPA) und die Deutsche Gesellschaft für Allergologie und Klinische Immunologie (DGAKI) sowohl ihre Verbundenheit zueinander als auch ihr Zusammenstehen für eine starke Allergologie in Deutschland.

Das Motto des DAK 2017 lautet „Gemeinsam die Allergologie stärken! Forschung-Fortbildung-Versorgung" und zeigt auf, worauf es uns bei dieser Tagung ankommt: Hohe Wissenschaft und neueste Erkenntnisse aus den allergologischen Laboren dieser Welt umsetzbar machen für Ihre praktische Fortbildung und die Versorgung unserer Patienten.

Mit den bewährten Kongressformaten - Plenarsitzungen, AllergoCompact und den Symposien un-

\section{Deutscher Allergiekongress auf einen Blick}

Veranstalter

_ Ärzteverband Deutscher Allergologen (AeDA)

_ Deutsche Gesellschaft für Allergologie und klinische Immunologie (DGAKI)

_ Gesellschaft für Pädiatrische Allergologie und Umweltmedizin (GPA)

Kongresstermin und -ort

5.-7. Oktober 2017

Kurhaus Wiesbaden

\section{Kongresspräsidenten}

Prof. Dr. Ludger Klimek, Wiesbaden

Prof. Dr. Wolfgang Wehrmann, Münster

\section{Anmeldung und Information}

Aktuelle Informationen sind im Internet unter www.allergiekongress.de zu finden. Die Anmeldung ist online möglich oder mit nebenstehendem Anmeldeformular per Fax. serer Partner aus der Industrie - werden wir die Vielfältigkeit der Allergologie präsentieren. Dafür lohnt es sich auch zu streiten: Das zeigen die Pround-Kontra-Debatten (Pro-Con-Debatten) in der AllergoArena in beeindruckender Weise. Wirklich handfest und praktisch wird es dann in einer Reihe interdisziplinärer Workshops in Form der AllergoActives zu sämtlichen allergologischen Themen.

Wiesbaden wurde wohl auch deshalb zu einem der beiden dauerhaften Austragungsorte des DAK erwählt, weil wir dort in den historischen Ballsälen des Kurhauses tagen dürfen. „Das schönste Kurhaus der Welt!", schwärmte Kaiser Wilhelm II bereits bei der Eröffnung zu seinen Ehren im Jahre 1907. Gemeinsam mit dem Kurpark, dem „Bowling Green“ und dem hessischen Staatstheater ist es eingebettet in die historischen Gebäude in Jugendstil, Barock und Klassizismus der Altstadt von Wiesbaden. Ein Kongress im Herzen der Stadt!

Sowohl Neueinsteiger als auch erfahrene Allergologen werden vom interaktiven und interdisziplinären Austausch beim 12. DAK profitieren. Sie finden hier ideale Bedingungen zum wissenschaftlichen und fachlichen Networking: Treffen Sie alte und neue Freunde, die mit Ihnen das Interesse an der Allergologie teilen. Wo sonst haben Sie die Gelegenheit, über die Grenzen des eigenen Fachs hinauszuschauen und praktische Erfahrungen in „Hands-on“Veranstaltungen auch außerhalb der eigenen Fachdisziplin zu sammeln? Nutzen Sie das vielfältige Programm des Kongresses und lassen Sie sich von der Allergologie neu begeistern!

In diesem Sinne würden wir uns freuen, Sie zum 12. DAK in Wiesbaden begrüßen zu dürfen.
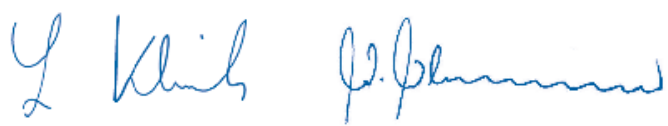